\title{
Norois
}

Environnement, aménagement, société

$250 \mid 2019$

Risque, littoral, paysage, port, géohistoire

\section{Sels et salines de l'Europe atlantique, Loïc Ménanteau}

\section{Michel Desse}

\section{(2) OpenEdition}

\section{Journals}

Édition électronique

URL : https://journals.openedition.org/norois/7467

DOI : 10.4000/norois.7467

ISBN : 978-2-7535-7873-9

ISSN : $1760-8546$

Éditeur

Presses universitaires de Rennes

Édition imprimée

Date de publication : 19 juin 2019

Pagination : 102-103

ISBN : 978-2-7535-7871-5

ISSN : 0029-182X

\section{Référence électronique}

Michel Desse, «Sels et salines de l'Europe atlantique, Loïc Ménanteau », Norois [En ligne], 250 | 2019, mis en ligne le 19 juin 2019, consulté le 08 janvier 2022. URL : http://journals.openedition.org/norois/7467 ; DOI : https://doi.org/10.4000/norois.7467

(c) Tous droits réservés 
géographie historique, cela correspond en fait à la géographie humaine actuelle (géographie urbaine, militaire, religieuse, agricole, industrielle...).

Mais ce n'est pas seulement un dictionnaire. À chaque fois, François de Dainville nous précise l'évolution cartographique. Comment étaient dessinés, gravés les fleuves, les lacs, les arbres, les villages au XVI ${ }^{\mathrm{e}}$ siècle, sur les premières cartes, et leurs évolutions vers plus d'abstraction. Il a réalisé des planches montrant ces évolutions. On peut rester de longs moments sur ces planches ultra-détaillées comme celles de la forêt ou des différents types d'arbres. Il nous explique par exemple les conventions cartographiques qui se mettent progressivement en place pour localiser des champs de bataille sur une carte. En plus de la localisation, on apporte une information supplémentaire en tournant de petites épées vers le haut quand le Prince a gagné une bataille et vers le bas quand celui-ci a perdu. Cette convention n'apparaît qu'au XVIII ${ }^{\mathrm{e}}$ siècle. L'éditeur a décidé de mettre toutes ses planches, ainsi que quelques extraits de cartes en bleu. C'est un choix discutable mais parfois cela rend la planche ou la carte plus lisible que le noir.

Cet ouvrage est donc à recommander à tout amoureux de la cartographie et de façon plus générale à toute personne ayant recours à des cartes anciennes. On peut penser par exemple aux candidats préparant le CAPES d'Histoire Géographie qui, à l'oral, bénéficieront à partir de juin 2019, d'un accès à Géoportail (www.geoportail.fr) et donc d'un accès aux cartes actuelles de l'institut Géographique National (IGN) mais également à la carte de Cassini ou d'État-major. L'ouvrage de François de Dainville devient alors utile et notamment, ses petites planches ainsi que son index des abréviations (p. 289-290) qui permettent de repérer des informations comme $\mathrm{M}^{\text {in }}$ ou $\mathrm{M}^{\mathrm{n}}$ qui correspondent à des moulins ou Riv. pour rivière et Sal. pour Saline... Comme quoi un ouvrage de 1964 peut être encore et toujours d'actualité.

Loïc Rivault

université de Rennes 2

\section{Ménanteau, Loïc, 2018. Sels et salines de l'Europe atlantique, Rennes, PUR, 504 p.}

Sels et salines de l'Europe atlantique sous la direction de Loïc Ménanteau est paru dans le cadre du projet Ecosal Atlantis. Cette contribution couvre tous les champs des sciences sociales et environnementales. Les 66 auteurs, universitaires et médiateurs culturels de la façade atlantique européenne du Portugal à l'Écosse contribuent à la richesse de l'ouvrage. Ce beau livre de 504 pages est remarquable par l'apport de connaissances sur les espaces et les sociétés salicoles mais aussi par la qualité de l'illustration provenant en partie des musées de l'Ouest atlantique. Cartes, peintures, photographies anciennes et affiches permettent d'ancrer cette aventure salicole dans le temps long. Certaines études de cas sont particulièrement illustrées comme les collections de salières ou la présentation des richesses de la biodiversité « du valle Salado de Anana » au pays Basque. La situation actuelle est, elle aussi couverte par une iconographie de très grande qualité permettant d'identifier les principales espèces de l'avifaune ou de mesurer l'évolution contemporaine des paysages et des pratiques d'acteurs. La cartographie toujours présente illustre les routes du sel à toutes les échelles géographiques.

L'ouvrage se divise en 4 parties. La première de près de 200 pages traite de «la production du sel et la géohistoire des salines de l'Écosse au détroit de Gibraltar ». L'approche archéologique de l'exploitation du sel y est particulièrement documentée aux travers d'études de cas multiscalaires sur le littoral breton, la baie du Mont Saint-Michel, la Loire atlantique, Batz et Guérande, Vigo ou encore dans les cuvettes salinières de Canto Marinho au Portugal et la large palette des méthodes de production. Dans la partie méridionale de l'Europe atlantique, la saliculture par évaporation solaire a été dominante alors qu'en Europe du Nord, les procédés de pré-concentration dominent. La Bretagne et le Royaume-Uni sont à la charnière de ces deux méthodes. Il en découle l'évolution historiques des paysages salicoles de Batz-Guérande, de la ria d'Aveiro, des 


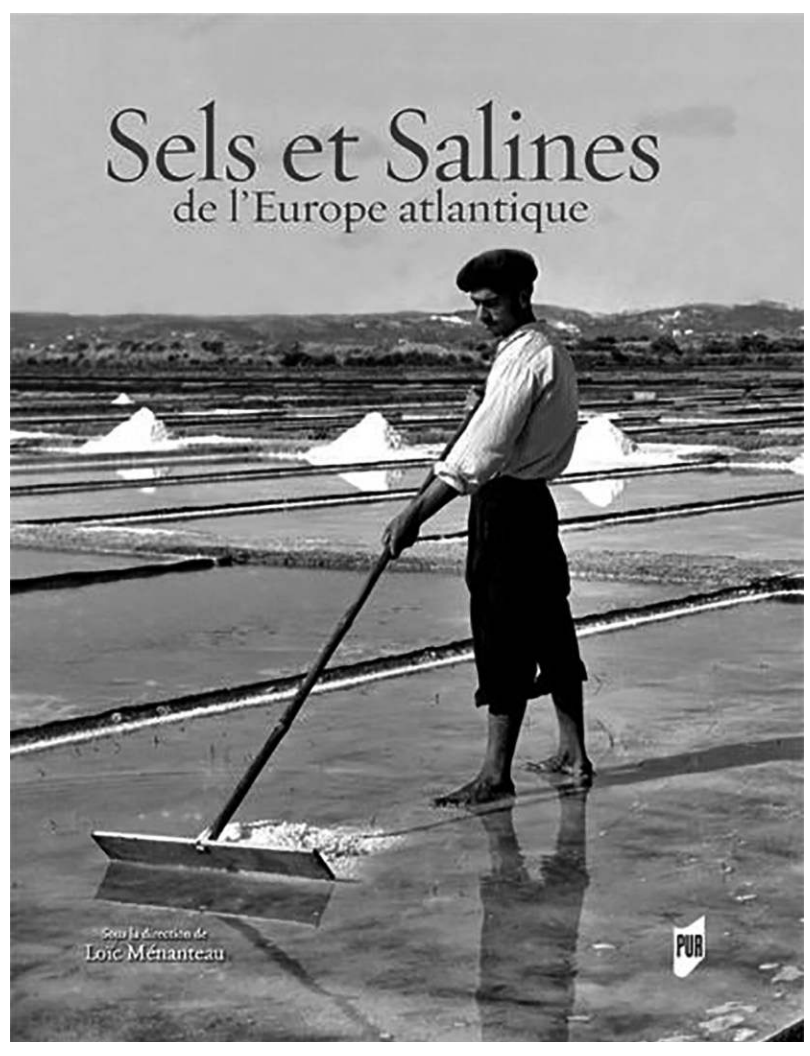

embouchures du Guadiana et du Guadalquivir ou encore de la baie de Cadix. Cette approche géohistorique permet de comprendre les différentes logiques d'appropriation et de développement des salines depuis le Moyen Âge et les régressions contemporaines à des fins agricoles ou aquacoles (Baie de Cadix). Enfin le petit patrimoine, cabanes, magasins à sels, roues hydrauliques n'est pas oublié dans sa diversité. On y retrouve cependant la même logique liée à la fonction et à la production.

La seconde partie d'une centaine de pages porte sur les usages du sel. Tout d'abord son commerce jusqu'à la Baltique au Moyen Âge, puis vers l'outreAtlantique avec l'exemple de Cadix qui exportait la moitié de sa production vers l'Argentine, l'Uruguay et le Sud du Brésil. Un chapitre est consacré aux salaisons pour la viande (exemple du Dorset [Royaume-Uni] dès l'âge du fer) et la conservation du poisson avec une étude des salaisons sur la côte du golfe ibérico-marocain de la haute antiquité à l'époque moderne. Logiquement, les moyens de transport s'adaptent aux produits et aux marchés : embarcations des marais de Guérande, ou barques à sel de la lagune d'Aveiro sans oublier le transport animal. Taxes et impôts sur le sel accompagnent cette économie et dessinent de véritables frontières économiques comme la région d'Ancenis entre gabelle et contrebande. L'approche des richesses environnementale est abordée dans la troisième partie. Ces espaces salicoles, adossés souvent à des zones humides, parfois abandonnés comme le Marais Breton se situent sur les principaux couloirs migratoires et abritent une avifaune riche et variée. Chaque espèce ne fait pourtant pas les mêmes haltes et chaque site diffère en fonction de sa superficie et des caractéristiques singulières de ses habitats qui constituent aussi des milieux contraignants pour les espèces terrestres avoisinantes.

Les marais salants en activité ou à l'abandon constituent souvent des espaces naturels protégés, dans le cadre de plans de gestion. Sites du réseau Natura 2000, protection au titre des directives oiseau et habitat, sites classés, réserves naturelles, parcs naturels régionaux et nationaux, réserves de biosphère constituent autant de modes de gestion différenciés.

La dernière partie montre l'intérêt écotouristique des sites salicoles atlantiques porteurs d'une histoire, de paysages et d'environnements particuliers. Du Royaume-Unis à l'Andalousie, 12 sites constituent la route "sel traditionnel, Route de l'Atlantique ", objectif du projet ECOSAL Atlantis. Cette route du sel comme d'autres routes touristiques en Europe insiste sur les bonnes pratiques de production et d'interprétation orientées vers un tourisme durable.

Sels et salines de l'Europe atlantique de Loïc Ménanteau permet de faire un point sur ces espaces particuliers, leurs activités et les sociétés qui marquent encore ces paysages qui ont pourtant parfois perdu leur fonction productive initiale entraînant de nouvelles pratiques et de nouveaux usages. Il constitue un ouvrage de référence par la richesse, la variété et la qualité de son iconographie.

Michel Desse Université de Nantes 